
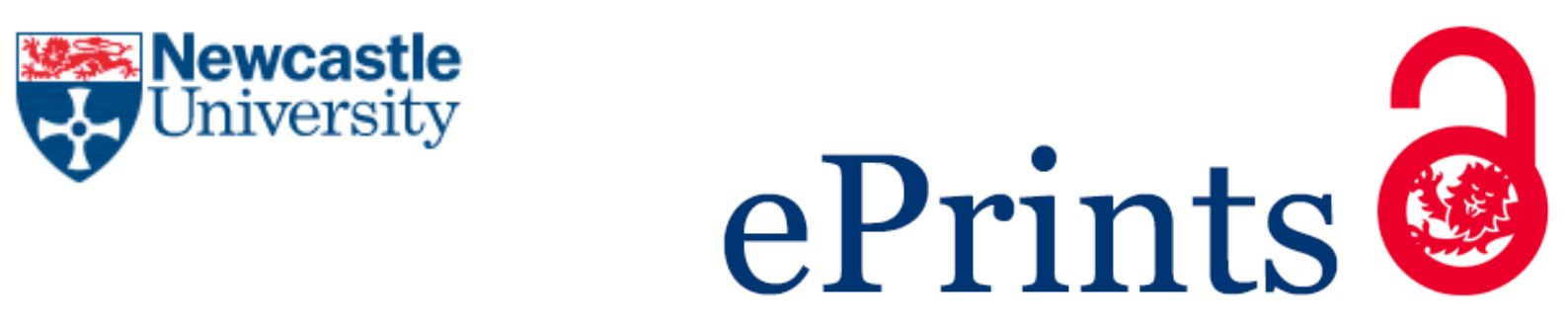

Lenthall R, McConachie N, White P, Clifton A, Rowland-Hill C.

BSNR Training guidance for mechanical thrombectomy.

Clinical Radiology 2017, 72(2), 175.e11-175.e18.

\title{
Copyright:
}

(C) 2017. This manuscript version is made available under the CC-BY-NC-ND 4.0 license

DOI link to article:

https://doi.org/10.1016/i.crad.2016.11.007

Date deposited:

$20 / 06 / 2017$

Embargo release date:

14 December 2017

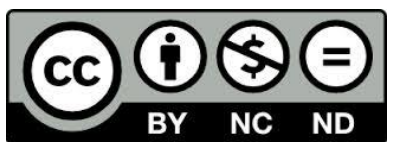

This work is licensed under a

Creative Commons Attribution-NonCommercial-NoDerivatives 4.0 International licence 


\section{BSNR training guidance for mechanical thrombectomy}

\section{d5 $\operatorname{Dr}_{2}$ R. Lenthall, Dr. N. McConachie, Prof. P. White, Dr. A. Clifton, 21 $\operatorname{Dr} . \mathrm{L}_{\mathrm{C}}$. Rowland-Hil[, UK Neurointerventional Group and British Society of Neuroradiologists}

Guidance for practitioners seeking training to participate in an acute ischaemic stroke thrombectomy service in the UK: a response to level- 1 evidence of the benefit of mechanical thrombectomy for patients with acute ischaemic stroke and proven large vessel occlusion.

Guidance produced by members of the UK Neurointerventional Group and British Society of Neuroradiologists on behalf of the Royal College of Radiology,

March 2016

\section{Preamble}

Following accumulating level-1 evidence of the benefit of timely mechanical thrombectomy (MT) for patients with acute ischaemic stroke (AIS) due to proven large vessel occlusion (LVO), there is an urgent need to train an additional cohort of practitioners to enable introduction of MT as a routine service in the UK. ${ }^{18}$

In the USA and Europe practitioners from different clinical backgrounds (radiology, neurology, neurosurgery, cardiology) can enter Interventional Neuroradiology (INR) training. This guidance proposes to create a pathway for practitioners (pre or post CCT) wishing to obtain RCRrecognised training to perform and participate in an acute stroke service in the UK.

This document draws on previous and current training guidance. It has been produced in response to an anticipated shortfall in service capacity and is intended to supplement, rather than replace current RCR training guidance.

\section{Introduction}

Recent clinical trials have shown that if eligible patients with acute stroke caused by LVO are rapidly treated with MT, their prospects for independent recovery are significantly improved. Successful management of patients with acute stroke is dependent on the swift, efficient function of a care pathway involving multiple pre-hospital and hospital services. A multi-societal consensus document describing service standards for practice in the UK has been published. ${ }^{18}$

NICE have reviewed the evidence for MT and have recently published updated guidance. ${ }^{23}$ This guidance supports the use of MT providing requirements for patient selection, operator training and service standards are adhered to. In this light there is need to define and train the clinicians who will help expand and deliver this service in the future.

Opening a blocked artery is a mechanical task common to multiple clinical specialties, however any clinician undertaking MT for patients with acute stroke will need detailed knowledge of clinical neurology, neuroanatomy and neurophysiology. They will require advanced radiological skills to evaluate neuroimaging studies and they will depend on broad case experience to interpret imaging findings in the clinical context and to select and manage patients appropriately.

Key objectives of training will include: developing a thorough understanding of neurological diseases, acquiring the range of technical skills required to manage neurovascular conditions, and refining the ability to make independent clinical decisions about timely, appropriate and 
safe interventional procedures (in relation to alternative non-interventional approaches).

In addition to having procedural technical skills and experience navigating catheter systems and thrombectomy devices in the cervical and intracranial circulation, operators will have to perform procedures under time pressure, frequently in challenging settings such as, difficult vascular access, patient movement and incomplete visualisation of the cerebral circulation.

After completing training, practitioners will be required to join teams providing a MT service and to contribute to on-call provision. Practitioners will need to maintain and refine their knowledge and skills, as evidence and technology evolves.

At current staffing levels, most INR services in the UK do not have sufficient resources to expand operating capacity to the level required to deliver a 24/7 MT service immediately. A crude estimate would require UK operator numbers to double (from 90 to 180) to enable robust on-call rotas to be established in existing INR centres. The RCR recommends a minimum $1 / 6$ rota frequency to provide a sustainable IR on-call rota. ${ }^{19}$ If UK trainee numbers were expanded to 20-25/year it would take $4-5$ years to train the required number of INRs (accounting for up-coming retirements).

Within current specialty training programs in the UK, there is only one subspecialty that provides training to perform MT (INR). The current training programme for INR takes six years. This is divided into two parts. The first three years is dedicated to training in core radiology skills. The fourth year is focused on acquiring advanced competencies in diagnostic neuroradiology and the fifth and sixth years are dedicated to INR training and refining neuroradiology skills. ${ }^{15}$

It is widely understood within the INR community that INR training provides sound knowledge and a broad grounding in technical skills, but that a consultant will continue to accumulate essential case-experience during the early years of their consultancy. This on-going formative process usually occurs with the close support and guidance of experienced consultant colleagues.

In order to expand capacity to provide a MT service at a national level in the UK, it will be necessary to expand the UK INR training program. To address the required scale of expansion and the clinical imperative to provide a MT service, it is also important to consider options for training a cohort of practitioners in an accelerated time frame. As part of this process, it is proposed that INR training will be made available to clinicians from different clinical backgrounds.

To deliver this training successfully, the background and competencies of individual trainees will be taken into account and the content of training will focus on the knowledge, skills and competencies that are essential to enable the practitioner to function independently as part of a team providing a MT service. ${ }^{14,15,17}$

In considering how to expand operator capacity to meet the anticipated demand for 24/7 MT, the subsequent issues should be accounted-for:

Practitioners should adhere to the principles of Good Medical Practice Where practitioners undertake invasive clinical procedures for the $1^{\text {st }}$ time, they should be properly trained and directly supervised. ${ }^{6,9}$
The term mechanical thrombectomy (MT) refers to a range of operative techniques that are tailored to specific clinical and anatomical scenarios. MT is not a single procedure that can be quickly learnt and applied in practice.

Any specialist contributing to provision of a MT service should have completed a RCR-recognised training process. Patients are entitled to know what training and what experience a clinician has,

Training must account for the specific clinical back- 2 ground and prior experience of the trainee.

Trainees must acquire all of the skills required to provide a MT service. This must include a comprehensive understanding of the significance of imaging findings in the context of the patient's clinical status. It is not good interventional practice for another clinician (neurologist, stroke physician) to select the patient and instruct the operator, simply as a technician, when to perform a MT procedure. $5,8,15,17,19,20$.

Qualified interventional radiologists and cardiologists have skill sets that exceed core radiology training and are equivalent to INR training in specific areas. However, trainers and trainees should be aware of the potential mismatch between technical competencies that may be acquired rapidly by clinicians with an interventional background and diseasespecific knowledge and clinical judgment, which are acquired over a longer time, with broad case- experience.

Industry-sponsored educational courses, web-based teaching and scenario practice using simulators all provide useful educational opportunities. Whilst some of these options may contribute towards acquisition of competence, they are not as-yet integrated into radiology training in the $\mathrm{UK}$, they are not recognised as qualifications by the RCR/ GMC and they are not equivalent to training delivered in a recognised UK training program. Good quality web- based or simulation-based training should be accounted-for and may become accreditable experience in the future (with a recommended limit of $10 \%$ of total required experience). ${ }^{5,9}$

The natural history of neurovascular diseases and the procedural complications related to INR practice can be associated with poor clinical outcomes, including severe disability and death. Operators will need the clinical and interpersonal skills to deal with poor patient outcomes, clinical error, duty of candour and investigation/complaint procedures.

Focused training to provide only one specific therapeutic procedure does not align with practice in any current UK specialty-training program, or with the anticipated process of credentialing. ${ }^{24}$

Many of the technical skills required to perform MT are common to other INR procedures. In order to acquire these skills efficiently, the trainee will participate in many varied INR procedures during their training.

Training to acquire MT skills will involve a significant full-time-equivalent commitment for individual trainees (due to the infrequent and unpredictable case referral rate for MT even in large, high-volume centres). As a result of the time commitment required to acquire the requisite case experience in stroke, the trainee will be in a position to acquire many of the skills required for the wider INR area of practice. 
There are two essential patient care pathways that will benefit from provision of a comprehensive 24/7 INR on-call service, namely, a 24/7 MT service for patients with AIS and the aneurysm coiling service for patients with ruptured brain aneurysms (best provided 7 days per week). Less frequently, patients referred by other acute surgical services will also benefit from an on-call INR service, including Ear Nose and Throat surgery (epistaxis and carotid blow-out) and Trauma services (blunt/penetrating cranio-cervical vessel injury). 2,13,18.

In this light, it would be advisable that any substantial national investment in a training process is optimised to enable the qualified operators to contribute to a comprehensive INR on-call service that can meet all of the urgent needs of the wider referred population. 2,13,18,19

In this preferred model, trainees will acquire the ability to provide a wider- range of INR procedures (rather than MT alone). The range of skill sets at completion of training would be determined by a number of individual and centrerelated factors (following curriculum based training).

For training purposes, a qualified (post-CCT) practitioner entering INR training consultant interventional radiologist or cardiologist) should be regarded as an experienced trainee. It is important that (however qualified) a trainee should not be in a position to decide when their INR training is completed (i.e. the process of determining qualification is independent from the student).

After completion of training, maintenance of clinical and technical skills will be essential. MT cannot be performed infrequently with the expectation that clinical outcomes will be equivalent to those achieved by experienced operators providing the entire INR area of practice in highvolume centres. ${ }^{18}$

This document does not set out a path for practitioners to train to perform MT alone, but this possibility is not specifically excluded. In a scenario where practitioners were trained to perform MT as their only intracranial vascular procedure, assuming a reasonable on-call rota (1:6 or better), the operators would need to work in an extremely high volume MT centre to maintain case- experience and competencies $(>240$ procedures per annum would requiring a centre-catchment population of over 2.2 million).

A model where practitioners were trained to perform MT only might divide training and service resources in INR. This might impair service development, limit staffing levels in INR units and limit staffing of 7-day services to treat patients with aneurysmal SAH. ${ }^{13}$

\section{Components and duration of INR training pre-CCT}

INR trainee ${ }^{14,15}$

\begin{tabular}{ll}
\hline Core radiology training & 36 months \\
Advanced neuroimaging and neurosciences & 12 months \\
INR training & 24 months \\
\hline
\end{tabular}

Interventional Radiology trainee

An IR trainee wishing to convert to INR would need a minimum of 2 years of training time in diagnostic and interventional neuroradiology (i.e. would need to commence INR training by the start of ST5).

\section{Non-radiology trainee wishing to convert to INR training}

As-yet, there is no RCR/GMC approved path for trainees from other clinical specialties to enter into fast-track radiology training. At-present, trainees can either retrain in radiology, or alternatively complete their specialty training and retrain post-CCT.

\section{Components and estimated duration of INR/ MT training post-CCT}

Diagnostic and INR training can run concurrently.

\section{Interventional radiologist post-CCT}

\begin{tabular}{ll}
\hline Advanced neuroimaging and neurosciences & 6 months \\
INR training & $12-18$ months \\
Total & $12-24$ months \\
\hline
\end{tabular}

\section{Diagnostic neuroradiologist post-CCT}

\begin{tabular}{ll}
\hline INR training & $18-24$ months \\
Total & $18-24$ months* \\
\hline
\end{tabular}

Cardiologist post-CCT

\begin{tabular}{ll}
\hline Core/advanced neuroimaging and neurosciences & 12 months \\
INR training & 24 months \\
Total & 24 months* \\
\hline
\end{tabular}

Neurologist or neurosurgeon post-CCT

\begin{tabular}{ll}
\hline Core/advanced neuroimaging & 12 months \\
INR training & 24 months \\
Total & 24 months* \\
\hline
\end{tabular}

See appendix 1 for duration of training in other countries.

The time-lines provided are estimates of training time required to acquire competencies (rather than time intervals that define recognition of competence). Competence may be achieved at a faster, or slower rate. Non- radiologists in the USA require a minimum 6 months of cognitive neuroscience training to credential for cervico-cerebral diagnostic/interventional procedures.,

It may be possible to acquire diagnostic and interventional skills in parallel (saving time). However, based on anticipated MT case referral rates to UK Neuroscience centres, it is unlikely that any trainee would gain sufficient interventional case experience in a training interval shorter than 18 months. 
The time-lines for estimated duration of INR/MT training post-CCT complement recommendations in a recent multi-society consensus document which stipulates a minimum of one year of clinical neuroscience and neuroimaging training prior to a year of dedicated INR training. ${ }^{26}$

It is anticipated that 'qualified' operators will join INR services and work in teams that enable/facilitate continued learning and acquisition of case experience in a supported environment (rather than commencing practice as single-handed operators attempting to establish new services). ${ }^{22}$

\section{Training Centre Requirements}

INR training can only be provided in a recognised neuroscience training- centre or network, by experienced INRs. ${ }^{22}$

Training centres should be recognised by the UKNG/ BSNR/RCR/GMC. ${ }^{22}$ A training centre must include a team of at-least two INRs: ${ }^{11,22}$

The training centre must perform a minimum of 100 cerebral neurointerventional cases/year ${ }^{1,16}$ (European minimum 150-200/year ${ }^{8,11}$ ) and the case-mix should include acute stroke (a high volume of stroke work is an implicit training requirement, but it is impractical to set a specific target before anticipated MT service development).

The training centre must involve the trainee in every aspect of patient care including:

Diagnostic neuroimaging reporting MDTs (especially neurovascular) Pre-operative assessment

Patient consent Procedural planning

Team sign-in (WHO check) INR procedure

Post-op ward round

Ward based care (including intensive care) Post-op/ surveillance imaging

Outpatient clinics

The training centre must ensure that the trainee participates in:

Basic and advanced neuroimaging services (protocolling, supervising and reporting a wide range of examinations) On-call service provision

Database activity and case registries Morbidity and mortality meetings Clinical audit

Clinical Research (whenever possible)

Interactions with clinical proctors and industry representatives

The training centre must support the following educational processes Provision of educational teaching sessions.

Trainee attendance at relevant educational meetings Educational supervision

Assessment of diagnostic imaging skills (IPIX) Assessment of technical skills (DOPS) Summative assessment (ARCP/equivalent)

\section{Curricular content for MT/INR training}

Overarching training requirements for diagnostic and interventional neuroradiology are outlined in the RCR documents 'Specialty Training Curriculum for Clinical Radiology' November 2015, ${ }^{14}$ and 'Subspecialty Training Curriculum for Interventional Radiology' November 2015, ${ }^{15}$ pages $18-25$.

The RCR curricula provide an overview of training. A more comprehensive description of the syllabus covered during INR training in the UK is provided in the BSNR document Interventional Neuroradiology Curriculum V7. ${ }^{17}$ Focused training to enable provision of an MT service would require thorough coverage of the specific curricular content in this document.

Previous BSNR/RCR guidance has not set explicit targets for training activity or documented procedure rates. There are potential disadvantages to setting targets, the achievement of which may not necessarily reflect an individual's competence. However, the following section outlines procedural activity levels suggested by training bodies within and outside the UK as guidance.

\section{Reporting diagnostic imaging studies}

A neuroradiology trainee typically reports a minimum of 40 mixed CT/MR cases per week resulting in a low estimate of 1600 cases in a year.

Published target activity levels for complex imaging reporting include:

\begin{tabular}{lll}
\hline Activity & Number & Reference \\
\hline MRI & 2000 & 4 \\
CTA & 50 & 9 \\
MRA & 50 & 9 \\
Perfusion studies & 25 & 9 \\
\hline
\end{tabular}

Minimum targets such as these are likely to be farexceeded, if the trainee/fellow participates in provision of a neuroimaging service and reports all of the imaging studies related to the interventional procedures that they are involved in.

The aim is to be able to report all CT/MRI imaging as it relates to neurovascular diseases and to be able to rapidly and independently discriminate imaging appearances of neurovascular disease from mimics/other pathology.

Trainees should arrange regular evaluation of their reporting skills (IPIX) and training supervisors should be aware of their trainees' reporting discrepancies.

\section{Cerebral catheter angiography}

Expertise in diagnostic cerebral angiography provides the foundation for safe and successful INR practice.

There is good evidence that fluoroscopy times, patient radiation dose and procedural complication rates decline with increased operator experience. ${ }^{5,9}$

There is good evidence that observer interpretive error declines as operator experience increases. ${ }^{5,9}$ 
Angiography during MT procedures:

Operators will need to correlate imaging appearances on non-invasive imaging (CTA, MRA) with imaging appearances at catheter angiography.

Operators will need to identify: anatomical variation, arterial pathology and the effects of altered flow.

Misinterpretation of appearances may result in erroneous decisions to withhold or apply specific measures, increasing patient risk.

Examples of published recommended minimum angiographic experience include:

\begin{tabular}{|c|c|c|}
\hline Institution & Experience & Reference \\
\hline RANZCR & 150 supervised & 4 \\
\hline $\begin{array}{l}\text { American Heart } \\
\text { Association et-al }\end{array}$ & 100 diagnostic angiograms & 5 \\
\hline $\begin{array}{l}\text { American College of } \\
\text { Cardiology }\end{array}$ & $\begin{array}{l}300 \text { diagnostic coronary } \\
\text { angiograms }\end{array}$ & 5,9 \\
\hline $\begin{array}{l}\text { American Academy of } \\
\text { Neurology }\end{array}$ & $\begin{array}{l}100 \text { supervised cerebral } \\
\text { angiograms }\end{array}$ & 5 \\
\hline RCR & 150 diagnostic angiograms & 6 \\
\hline British Cardiac Society & $\begin{array}{l}200 \text { diagnostic coronary } \\
\text { angiograms }\end{array}$ & 6 \\
\hline $\begin{array}{l}\text { American College of } \\
\text { Radiology }\end{array}$ & $\begin{array}{l}50 \text { supplemented by other } \\
\text { experience }\end{array}$ & 9 \\
\hline ACGME INR training & 100 before INR training* & $9, *$ \\
\hline SIR & 200 selective angiograms & 9 \\
\hline UKNG/BSNR 2016 & $\begin{array}{l}100 \text { (can include non-MT } \\
\text { INR cases) }\end{array}$ & This document \\
\hline
\end{tabular}

* mandated requirement prior to entering INR training and not altered by prior angiographic experience in any other vascular territory.

\section{Carotid angioplasty and stenting}

\begin{tabular}{lll}
\hline Institution & Experience & Reference \\
\hline RANZCR & 30 (15 as primary) & 4 \\
RCR & 15 cases/year & 6 \\
UKNG/BSNR 2016 & 10 cases per year* & This document \\
\hline
\end{tabular}

* This recommendation only applies to practitioners performing a wide range of INR procedures. In this setting it would be reasonable to include vertebral artery or intracranial artery angioplasty and stenting.

\section{Other stenting experience}

\begin{tabular}{|c|c|c|}
\hline Institution & Experience & Reference \\
\hline RANZCR & 50 peripheral stents (primary operator) & 4 \\
\hline $\begin{array}{l}\text { American College } \\
\text { of Cardiology }\end{array}$ & 100 peripheral angioplasty & 5 \\
\hline $\begin{array}{l}\text { American College } \\
\text { of Cardiology }\end{array}$ & $\begin{array}{l}250 \text { supervised coronary stent } \\
\text { procedures }\end{array}$ & 5,9 \\
\hline RCR & 130 peripheral angioplasty & 6 \\
\hline RCR & 50 peripheral stents & 6 \\
\hline
\end{tabular}

\section{INR operative procedures}

These include primarily the treatment of ruptured and unruptured intracranial aneurysms. See curricula for description of other related procedures. . $^{15,17}$

\begin{tabular}{lll}
\hline Institution & Experience & Reference \\
\hline WFITN & 160 procedures (30 primary operator) & 7,8 \\
UEMS & 150 procedures (50 primary operator) & 11 \\
ANZSNR/ANZAN/NSA & 60 aneurysms (30 primary operator & 16 \\
& 20 angioplasty or stent procedures & \\
& 20 particulate embolisations \\
& 10 liquid embolisations & \\
\end{tabular}

\section{Arterial recanalisation procedures (MT)}

\section{Considerations:}

This procedure demands rapid arterial access, rapid navigation of a guide catheter system to a stable position, accurate assessment of arterial anatomy, rapid navigation of a microcatheter system and or suction thrombectomy catheter intracranially and safe deployment and retrieval of a stentriever.

The operator must appreciate what equipment is best suited to the anatomy and the procedure, where and how to navigate blindly and what manipulation the arterial tree will tolerate (cerebral vessels are thin-walled, mobile/ deformable, angulated and take multiple variable branching patterns).

The operator must be equipped with the necessary technical and decision- making skills to be: able to continuously re-assess progress, able to modify the strategy where appropriate, able to decide when an effective treatment has been achieved and able to detect and manage complications promptly.

In the clinical setting (often post IV thrombolysis) the consequences of vessel perforation, vessel dissection or rupture (due to inadvertent navigation into a perforator, small pial arterial branch, or aneurysm), may be fatal. Inefficient technique will also increase procedural time, reducing benefit and increasing the risk of complications such as clot embolisation to another vessel territory.

Minimum recommended case experience:

\begin{tabular}{lll}
\hline Institution & Experience & Reference \\
\hline SIR & $\begin{array}{l}\text { 200 selective angiograms of which } 50 \\
\text { should be cervico-cerebral } \\
30 \text { procedures using microcatheters } \\
\text { and micro guidewires } \\
5 \text { stroke lysis cases under the (remote) } \\
\text { supervision of a proctor who has } \\
\text { performed at-least 10 cases }\end{array}$ & \\
& $\begin{array}{l}1 \text { year of INR fellowship with the full } \\
\text { range of INR procedures }\end{array}$ & 10 \\
AAN, AANS/CNS, & \\
SNIS, & $\begin{array}{l}100 \text { cerebral angiograms } \\
\text { SVIN }\end{array}$ & 10 \\
AAN, AANS/CNS, intracranial microcatheter \\
SNIS, \\
SVIN & $\begin{array}{l}\text { navigations 10 mentored } \\
\text { stroke therapies* }\end{array}$ & \\
UKNG/BSNR & 30 MT procedures & $\begin{array}{l}\text { This } \\
\text { document }\end{array}$ \\
\hline
\end{tabular}

* the advisory figures provided relate primarily to intra-arterial thrombolysis (2009) and are not directly comparable to current MT techniques (which would be associated with higher risks in less experienced hands).

Both quoted publications ${ }^{9,10}$ relate to practice in the USA prior to 2010. 


\section{Evidence of satisfactory completion of training for post-CCT trainees}

Post-CCT trainees will need to provide the following evidence to enable recognition of suitable training experience by the UKNG/BSNR/RCR:

Attendance record at the training institution(s).

Documented reporting activity for basic and advanced neuroimaging studies Evidence that diagnostic performance is equivalent to radiology SpRs at the equivalent level of training (IPIX $>5 \%$ per modality).

Log book documentation of the following minimum activity levels (half of each interventional activity should be as lead operator (DOPS $>10 \%$ per procedure).

\begin{tabular}{|c|c|}
\hline Activity & Number \\
\hline Cranio-cervical CTA reporting & 150 \\
\hline CTASI evaluation, or perfusion studies & 50 \\
\hline Cranio-cervical MRA reporting & 150 \\
\hline Cerebral catheter angiography (overlap allowed) & 100 \\
\hline $\begin{array}{l}\text { Navigation of guide catheter systems } \\
\text { (cervical and intracranial) }\end{array}$ & 100 \\
\hline $\begin{array}{l}\text { Intracranial navigation of } \\
\text { microcatheter/microwire systems }\end{array}$ & 100 \\
\hline Coiling of an intracranial aneurysm & 80 \\
\hline $\begin{array}{l}\text { Device-assisted coiling techniques } \\
\text { (balloon, stent, other) }\end{array}$ & 40 \\
\hline MT for AIS & 30 \\
\hline $\begin{array}{l}\text { Carotid, vertebral or intracranial } \\
\text { angioplasty/stent for atheroma }\end{array}$ & 10 \\
\hline $\begin{array}{l}\text { MT for AIS (in the event that stand-alone } \\
\text { training to perform } \\
\text { MT is supported) }\end{array}$ & 80 \\
\hline
\end{tabular}

Evidence of participation in M\&M and clinical audit meetings.

Evidence of attendance at relevant educational meetings and satisfactory CPD.

Supportive reports will be required from three UK training supervisors at least two of whom will be INRs actively practicing in the NHS. ${ }^{6,9,11,16}$ Training supervisors will be required to confirm that the trainee/fellow:

Reports basic and advanced neuroimaging studies relevant to INR practice competently (equivalent discrepancy rates)

Selects patients for procedures appropriately Communicates well with the patient and family members Performs identified INR procedures safely and effectively Recognises and manages procedural complications Recognises procedural and personal limits

Integrates well with teams and clinical services

Has the ability to admit to mistakes and learn from them

Training targets will inevitably excite debate, however the task of setting clear standards in training is likely to be less challenging than the task of raising such standards post-hoc, once clinical practices are established.

\section{Underperformance}

Concerns relating to underperformance of a pre-CCT trainee would be worked-through following established policies/procedures.

Given the resource implications of INR training for a post-CCT practitioner, any concerns about aptitude or underperformance should be raised promptly.

It is anticipated that training and review of progress for practitioners who are post-CCT (IR/cardiologist/physician) will mirror the processes for radiology trainees. Administration of the process will be the joint responsibility of the trainer and trainee.

The approach to underperformance or a deteriorating relationship between trainer and trainee would follow local training program and Trust policies/procedures.

\section{Maintenance of skills}

Any practitioner performing MT must work in a centre that has direct access to multidisciplinary care with cover from:

Stroke Services

Anaesthetics

Neuro Critical Care

Neurosurgery

Vascular Surgery

The centre must have $24 / 7$ provision of CT/CTA and DSA angiography/interventional facilities, ${ }_{\text {, }}^{4,18,22}$

In order to justify the personal and institutional investment involved in undertaking training to perform INR procedures, it is essential that qualified practitioners work in institutions with a sufficient case-referral rate to maintain skills in clinical practice. ${ }^{18,21}$

The operator must perform no less than 40 intracranial neurovascular procedures/year (minimum 1 per working week, excluding diagnostic catheter angiography and isolated carotid artery stenting) to maintain competencies.

Examples of minimum criteria for maintenance of competence.

\begin{tabular}{lll}
\hline Institution & Experience & Reference \\
\hline RANZCR & 25 selective supra-aortic angiograms/year & 4 \\
RCR & 15 carotid stent procedures/year & 6 \\
UEMS & $\begin{array}{l}\text { Practicing INR > 80\% of time and meeting } \\
\text { CME/CPD requirements }\end{array}$ & 11 \\
ANZSNR/ANZAN/ & 100 INR cases/3 years & 16 \\
NSA & B0 intracranial vascular procedures/year & $\begin{array}{l}\text { This } \\
\text { document }\end{array}$ \\
& &
\end{tabular}

The implications of failure to maintain case experience following training include:

De-skilling (whole-team, not just the INR) Prolonged treatment times (patient harm) Complications, loss of confidence (giving up INR) Retraining needs

Wasting limited training resources 


\section{Immediate considerations for the UKNG, BSNR, BSIR, RCR}

\section{Training}

Approval of proposed training pathways for INR/MT.

RCR recognition of INR training centres (including assessment of equivalence of international training posts?).

Improve access to INR training.

Expansion of INR training posts, ideally to 20 for the next 5 years Establishment of the training post numbers required to support future consultant posts (10/year).

\section{Qualification}

No change to FRCR or CCT for radiology trainees.

RCR-approved process for recognition of completion of INR training (for post- CCT doctor undertaking INR training). (A training certificate?)

Post-qualification surveillance (Discuss how this might be regulated)-Annual verification of maintenance of competence via case-load documentation.

Annual reporting of quality standards: recanalization rates, ENT rate, $\mathrm{SICH}$ rate. ${ }^{26}$

Annual reporting of mortality/outcome data. ${ }^{6}$

Options: National audit UKNG/BSNR vs. process overseen by RCR equivalent to CPD registration. Site visits if thresholds not met?

\section{Mobilisation of resources}

Multi-society sign-up to training guidance Deanery support to expand training posts.

NHS-Trust support to enable qualified clinicians to enter INR training.

\section{Markers of success}

Expansion of existing teams and INR services Provision of on-call services.

Improved patient access to INR services National growth of MT case numbers.

Enable development of new services where improved patient access not geographically possible.

\section{Uncited reference}

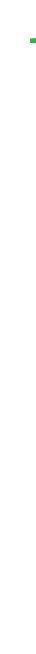

\section{Appendix 1}

Recommended duration of core neuroscience training

\begin{tabular}{lll}
\hline Location & Duration (months) & Reference \\
\hline USA & 6 & $5,9,10$ \\
Europe & 12 & 11,25 \\
\hline
\end{tabular}

Recommended duration of diagnostic neuroradiology training

\begin{tabular}{lll}
\hline Location & Duration (months) & Reference \\
\hline Australasia & 12 & 4 \\
USA & 12 & 5 \\
Europe & 12 & $7,8,25$ \\
UK & $12-24$ & 14 \\
\hline
\end{tabular}

Recommended duration of INR training

\begin{tabular}{lll}
\hline Location & Duration & Reference \\
\hline USA & 12 & 5 \\
Europe & 24 & $7,8,25$ \\
Europe & 30 & 11 \\
UK & 24 & 15 \\
Australasia & 24 & 16 \\
\hline
\end{tabular}

In Europe, the UEMS Training Charter indicates that physicians from all specialty backgrounds can be trained in INR. In order to ensure that clinicians from different backgrounds receive consistent training, the Training Charter is based on 4 years full-time training including: 1 year of clinical neuroscience, 1 year of diagnostic neuroradiology and 2 years of INR training. 25

\section{References}

1. Training in Interventional Neuroradiology. Molyneux A on behalf of the UKNG. November 1996

2. Interventional vascular radiology and interventional neurovascular radiology. NCEPOD. November 2000

3. Effective Neuroradiology. Guidelines for safe and effective practice. BSNR 2003.

4. Training requirements in interventional neuroradiology (INR) procedures. RANZCR_ANZSNR_IRSA Guidelines November 2004.

5. Connors III JJ et al. Training, competency and credentialing standards for diagnostic cervico- cerebral angiography, carotid stenting and cerebrovascular intervention etc. Neurology 2005;64:190-8.

6. BFCR(06)6 - Advice from the Royal College of radiologists concerning training for carotid artery stenting (CAS). October 2006

7. Richling $B_{1}$ et al. Standards of training in endovascular neurointerventional therapy. Acta Neurochir (Wein) 2007;149:613-6.

8. Interventional Neuroradiology Training Charter. WFITN Executive Committee. Interventional Neuroradiology 2009;15:11-5.

9. Training Guidelines for Intra-arterial Catheter-Directed Treatment of Acute Ischaemic Stroke. A statement from a special Writing Group of the society of interventional radiology (SIR). J Vasc Interv Radiol 2009;20:1507-22.

10. Meyers PM, et al. Performance and training standards for endovascular ischaemic stroke treatment. J NeuroInterv Surg 2009;1:10-2.

11. UEMS recommendations for acquiring 'Particular competence' in endovascular interventional neuroradiology - INR. August 2010

12. European curriculum and syllabus for interventional radiology. March 2013, CIRSE 
13. Managing the Flow? A review of the care received by patients who were diagnosed with an aneurysmal subarachnoid haemorrhage. NCEPOD 2013.

14. STC for Clinical Radiology. November 2015, RCR

15. Sub-specialty training curriculum for interventional radiology. November 2015, RCR

16. Conjoint Committee Guidelines for recognition of training in INR (ANZSNR, ANZAN, NSA)

17. BSNR Interventional Neuroradiology Curriculum V7, 2016. Lenthall R on behalf of the BSNR TESC

18. Standards for providing safe acute ischaemic stroke thrombectomy services. White PM et-al. BASP, BSNR, NACCS under oversight of ISWP

19. Standards for providing a 24 hour interventional radiology service. $R C R$ 2008.
20. Provision of Interventional Radiology services. The RCR in collaboration with the BSIR. 2012

21. Atkinson S. Quality domains. RCP definition of quality, Clinical Medicine 2010;10(6):537-9.

22. Safe Neuroradiology 2012. BSNR

23. Mechanical clot retrieval for treating acute ischaemic stroke. IPG 548. NICE Feb 2016.

24. http://www.gmc- uk.org/Introducing_Regulated_Credentials_Consul tation_W_form_FINAL_dist ributed.pdf_61589419.pdf

25. ESMINT statement regarding the UEMS training Charter for interventional neuroradiology. EJMINT Editorial 2013:1327000117.

26. Training Guidelines for Endovascular Ischaemic Stroke Intervention: An international multi-society consensus document. EJMINT Editorial 2016:1607000288. 